\title{
Research Article \\ Synchronization of Complex Networks with Time-Varying Delayed Dynamical Nodes via Pinning Control
}

\author{
Shuguo Wang, ${ }^{1,2}$ Hongxing Yao, ${ }^{2}$ and Qiuxiang Bian ${ }^{2,3}$ \\ ${ }^{1}$ Department of Mathematics and Physics, Hohai University, Changzhou Campus, Jiangsu, \\ Changzhou 213022, China \\ ${ }^{2}$ Faculty of Science, Jiangsu University, Jiangsu, Zhenjiang 212013, China \\ ${ }^{3}$ Faculty of Mathematics and Physics, Jiangsu University of Science and Technology, Jiangsu, \\ Zhenjiang 212013, China
}

Correspondence should be addressed to Shuguo Wang,wsg97@163.com

Received 17 August 2011; Accepted 19 October 2011

Academic Editor: Shengyong Chen

Copyright (c) 2012 Shuguo Wang et al. This is an open access article distributed under the Creative Commons Attribution License, which permits unrestricted use, distribution, and reproduction in any medium, provided the original work is properly cited.

This paper investigates the pinning synchronization of nonlinearly coupled complex networks with time-varying coupling delay and time-varying delay in dynamical nodes. Some simple and useful criteria are derived by constructing an effective control scheme and adjusting automatically the adaptive coupling strengths. To validate the proposed method, numerical simulation examples are provided to verify the correctness and effectiveness of the proposed scheme.

\section{Introduction}

In the past few years, the analysis and controllability of complex networks have attracted lots of attention [1-5]. One of the main reasons for that is its wide applications in biology [6], physics, and engineering [7]. So far, many different types of synchronization have been investigated, such as complete synchronization [8], generalized synchronization [9], phase synchronization [10], lag synchronization [11], projection synchronization [12, 13], and so forth. Meanwhile, many control approaches have been developed to synchronize complex networks such as adaptive control [14], pinning control [15, 16], impulsive control [17-20] intermittent control [21, 22], and so on.

For the complexity of the dynamical network, it is difficult to realize the synchronization by adding controllers to all nodes, such as [23]. To reduce the number of the controllers, a natural way is using pinning control method. Guo et al. [24] investigated the 
global synchronization of the complex networks with nondelayed and delayed coupling based on the pinning controllers. We can obtain some sufficient conditions for the global synchronization by adding linear and adaptive feedback controllers to some part of nodes from his woks. In [25], the authors investigated pinning control for linearly coupled networks and found that one can pin the coupled networks by introducing fewer locally negative feedback controllers. They also compared two different pinning strategies: randomly pinning and selective pinning based on the connection degrees and found out that the pinning strategy based on highest connection degree has better performance than totally randomly pinning. In [26], the authors pinned the complex network to the synchronization manifold by controlling one single node. And in [27], the authors investigated the pinning synchronization of delayed dynamical networks via periodically intermittent control. However, not much has been done on the synchronization of nonlinearly coupled complex networks with time-varying coupling delay and time-varying delay in dynamical nodes by pinning part of nodes and adjusting time-varying coupling strengths.

Motivated by the above discussions, in this paper, we work on the pinning synchronization of nonlinearly coupled complex networks with time-varying coupling delay and time-varying delay in dynamical nodes. The main contributions of this paper are threefold. (1) This paper deals with the synchronization problem for nonlinearly coupled complex networks with time-varying coupling delay and time-varying delay in dynamical nodes. The method used is adaptive pinning controlling method. By adjusting time-varying coupling strengths, some sufficient conditions for the synchronization are derived by constructing an effective control scheme, which are different from the methods used in [28]. (2) By using the Lyapunov stability theorem and a linear matrix inequality (LMI), we prove that the dynamical network can be made to be synchronous with one isolated node by adding controllers to only a small subset of the nodes. Compared with some similar designs, our pinning adaptive controllers are very simple. In addition, the pinning nodes can be randomly selected. It indeed provides some new insights for the future practical engineering design. (3) The Synchronization criteria are independent of time delay. Numerical examples are also provided to demonstrate the effectiveness of the theory.

The rest of this paper is organized as follows. The network model is introduced, and some necessary definitions, lemmas, and hypotheses are given in Section 2. The synchronization of the coupled complex networks is discussed in Section 3. Examples and their simulations are obtained in Section 4. Finally, conclusions are drawn in Section 5.

\section{Networks Models and Mathematical Preliminaries}

\subsection{Model Description}

The network with time-varying coupling delay and adaptive coupling strengths can be described by

$$
\begin{aligned}
\dot{x}_{i}(t)= & f\left(t, x_{i}(t), x_{i}(t-\tau(t))\right)+\sigma(t) \sum_{j=1}^{N} a_{i j} H_{1}\left(x_{j}(t)\right) \\
& +c(t) \sum_{j=1}^{N} b_{i j} H_{2}\left(x_{j}(t-\tau(t))\right), \quad i=1,2, \ldots, N,
\end{aligned}
$$


where $x_{i}(t)=\left(x_{i 1}(t), x_{i 2}(t), \ldots, x_{i n}(t)\right)^{T} \in R^{n}$ is the state vector of the $i$ th dynamical node, $f: R^{+} \times R^{n} \rightarrow R^{n}$ is an vector value function, $\tau(t)>0$ is time-varying delay, $\sigma(t)>0$ and $c(t)>0$ are time-varying coupling strengths, $H_{1}(\cdot)$ and $H_{2}(\cdot)$ are nonlinear functions, and $A=\left(a_{i j}\right)_{N \times N}$ and $B=\left(b_{i j}\right)_{N \times N}$ are the weight configuration matrices; if there is a connection from node $i$ to node $j(j \neq i)$, then, $a_{i j}=a_{j i}>0, b_{i j}=b_{j i}>0$, otherwise, $a_{i j}=a_{j i}=0$, $b_{i j}=b_{j i}=0$, and the diagonal elements of matrix $A, B$ are defined by

$$
a_{i i}=-\sum_{j=1, j \neq i}^{N} a_{j i}, \quad b_{i i}=-\sum_{j=1, j \neq i}^{N} b_{j i}, \quad i=1,2, \ldots, N .
$$

\subsection{Mathematical Preliminaries}

Definition 2.1. Assume that $s(t) \in R^{n}$ is any smooth dynamics. The controlled complex network (2.1) is said to be synchronized on to the homogeneous state $s(t)$ if the solution satisfies $\lim _{t \rightarrow \infty}\left\|x_{i}(t)-s(t)\right\|=0$ for any initial conditions.

Let $C\left([-\tau, 0], R^{n}\right)$ be the Banach space of continuous functions that map the interval $[-\tau, 0]$ into $R^{n}$ with norm $\|\phi\|=\sup _{-\tau<\theta<0}\|\phi(\theta)\|$. The initial conditions of the functional differential equation (2.1) are given by $x_{i}(\bar{t})=\phi_{i}(t) \in C\left([-\tau, 0], R^{n}\right)$. It is assumed that (2.1) has a unique solution for these initial conditions.

Suppose that $s(t)$ is a solution of the uncoupled system $\dot{s}(t)=f(s(t))$. In order to pin the system (2.1) onto the synchronization manifold $(s(t), s(t), \ldots, s(t))$, we will add the controllers $u_{i}(t)=k_{i}\left(H_{1}\left(x_{i}(t)\right)-H_{1}(s(t))\right)$ to a certain selection of the nodes where $k_{i}$ will be

defined in the following section. Without loss of generality, we add the controllers to the first $l$ nodes $(0 \leq l \leq N)$ so that we have

$$
\begin{aligned}
\dot{x}_{i}(t)= & f\left(t, x_{i}(t), x_{i}(t-\tau(t))\right)+\sigma(t) \sum_{j=1}^{N} a_{i j} H_{1}\left(x_{j}(t)\right) \\
& +c(t) \sum_{j=1}^{N} b_{i j} H_{2}\left(x_{j}(t-\tau(t))\right)-u_{i}(t), \quad i=1,2, \ldots, l, \\
\dot{x}_{i}(t)= & f\left(t, x_{i}(t), x_{i}(t-\tau(t))\right)+\sigma(t) \sum_{j=1}^{N} a_{i j} H_{1}\left(x_{j}(t)\right) \\
& +c(t) \sum_{j=1}^{N} b_{i j} H_{2}\left(x_{j}(t-\tau(t))\right), \quad i=l+1, l+2 \cdots N .
\end{aligned}
$$

Noting that $e_{i}(t)=x_{i}(t)-s(t)$, we have

$$
\begin{aligned}
\dot{e}_{i}(t)= & \tilde{f}\left(t, x_{i}(t), x_{i}(t-\tau(t))\right)+\sigma(t) \sum_{j=1}^{N} a_{i j} H_{1}\left(x_{j}(t)\right) \\
& +c(t) \sum_{j=1}^{N} b_{i j} H_{2}\left(x_{j}(t-\tau(t))\right)-u_{i}(t), \quad i=1,2, \ldots, l,
\end{aligned}
$$




$$
\begin{aligned}
\dot{e}_{i}(t)= & \tilde{f}\left(t, x_{i}(t), x_{i}(t-\tau(t))\right)+\sigma(t) \sum_{j=1}^{N} a_{i j} H_{1}\left(x_{j}(t)\right) \\
& +c(t) \sum_{j=1}^{N} b_{i j} H_{2}\left(x_{j}(t-\tau(t))\right), \quad i=l+1, l+2 \cdots N,
\end{aligned}
$$

where $\tilde{f}\left(t, x_{i}(t), x_{i}(t-\tau(t))\right)=f\left(t, x_{i}(t), x_{i}(t-\tau(t))\right)-f(t, s(t), s(t-\tau(t)))$.

We now introduce some definitions, assumptions, and lemmas that will be required throughout this paper.

Lemma 2.2 (see [29]). Assuming that $A=\left(a_{i j}\right) \in R^{N \times N}$ satisfies the following conditions:

(1) $A=\left(a_{i j}\right) \in R^{N \times N}, a_{i j}=a_{j i}>0, a_{i i}=-\sum_{j=1, j \neq i}^{N} a_{i j}, i=1,2, \ldots, N$,

(2) $A$ is irreducible.

Then we have that

(i) the real parts of the eigenvalues of $A$ are all negative except an eigenvalue 0 with multiplicity 1.

(ii) A has a right eigenvector $(1,1, \ldots, 1)^{T}$ corresponding to the eigenvalue 0 .

Lemma 2.3 (see [24]). If $G=\left(g_{i j}\right)_{N \times N}$ is an irreducible matrix that satisfies $g_{i j}=g_{j i} \geq 0$ for $i \neq j$, and $g_{i i}=-\sum_{j=1, i \neq j}^{N} g_{i j}$, for $i=1,2, \ldots, N$, then all the eigenvalues of the matrix

$$
\tilde{G}=\left(\begin{array}{ccc}
g_{11}-\varepsilon_{1} & \cdots & g_{1 N} \\
\vdots & \ddots & \vdots \\
g_{N 1} & \cdots & g_{N N}-\varepsilon_{N}
\end{array}\right)
$$

are negative, where $\varepsilon_{1}, \varepsilon_{2}, \ldots, \varepsilon_{N}$ are nonnegative constants and $\varepsilon_{1}, \varepsilon_{2}, \ldots, \varepsilon_{N}>0$.

Lemma 2.4 (see [30]). For any two vectors $x$ and $y$, a matrix $S>0$ with compatible dimensions, one has: $2 x^{T} y \leq x^{T} S x+y^{T} S^{-1} y$.

Lemma 2.5 (see [31]). Let $Q$ and $R$ be two symmetric matrices, and matrix $S$ has suitable dimension. Then

$$
\left(\begin{array}{cc}
Q & S \\
S^{T} & R
\end{array}\right)<0
$$

if and only if both $R<0$ and $Q-S R^{-1} S^{T}<0$. 
Lemma 2.6 (see [32]). For the vector-valued function $f\left(t, x_{i}(t), x_{i}(t-\tau(t))\right)$, assuming that there exist positive constants $\gamma>0, \theta>0$ such that $f$ satisfies the semi-Lipschitz condition

$$
\begin{aligned}
& \left(x_{i}(t)-y_{i}(t)\right)^{T}\left(f\left(t, x_{i}(t), x_{i}(t-\tau(t))\right)-f\left(t, y_{i}(t), y_{i}(t-\tau(t))\right)\right) \\
& \quad \leq \gamma\left(x_{i}(t)-y_{i}(t)\right)^{T}\left(x_{i}(t)-y_{i}(t)\right)+\theta\left(x_{i}(t-\tau(t))-y_{i}(t-\tau(t))\right)^{T}\left(x_{i}(t-\tau(t))-y_{i}(t-\tau(t))\right),
\end{aligned}
$$

for all $x, y \in R^{n}$ and $t \geq 0, i=1,2, \ldots N$.

Assumption 2.7 (see [9] (Global Lipschitz Condition)). Suppose that there exist nonnegative constants $\alpha, \beta$, for all $t \in R_{+}$, such that for any time-varying vectors $x(t), y(t) \in R^{n}$

$$
\left\|H_{1}(x)-H_{1}(y)\right\| \leq \alpha\|x-y\|, \quad\left\|H_{2}(x)-H_{2}(y)\right\| \leq \beta\|x-y\|,
$$

where || || denotes the 2 norm throughout the paper.

Assumption 2.8 (see [9]). $\tau(t)$ is differential function with $0 \leq \dot{\tau}(t) \leq \varepsilon \leq 1$. Clearly, this assumption is certainly to ensure that the delay $\tau(t)$ is constant.

\section{Main Results}

In this section, a control scheme is developed to synchronize a delayed complex network with time-varying delay dynamical nodes to any smooth dynamics $s(t)$. The local controllers are designed as follows:

$$
u_{i}(t)=k_{i}\left(H_{1}\left(x_{i}(t)\right)-H_{1}(s(t))\right),
$$

we let

$$
\widetilde{A}=\left(\begin{array}{cccccc}
a_{11}-\frac{k_{1}}{\mu_{1}} & \ldots & a_{1 l} & a_{1, l+1} & \ldots & a_{1 N} \\
\vdots & \ddots & \vdots & \vdots & \vdots & \vdots \\
a_{l 1} & \ldots & a_{l l}-\frac{k_{l}}{\mu_{1}} & a_{l, l+1} & \ldots & a_{l N} \\
a_{l+1,1} & \ldots & a_{l+1, l} & a_{l+1, l+1} & \ldots & a_{l+1, N} \\
\vdots & \ddots & \vdots & \vdots & \vdots & \vdots \\
a_{N 1} & \ldots & a_{N l} & a_{N, l+1} & \ldots & a_{N N}
\end{array}\right)
$$


where $k_{1}, k_{2}, \ldots, k_{l}$ are positive constants. It follows from Lemma 2.3 that if $A$ is symmetric and irreducible, then $\tilde{A}$ is negative definite. We can therefore prove the following theorem. For convenience in later use, we denote

$$
\begin{gathered}
\tilde{x}_{i}(t)=\left(x_{1 i}(t), x_{2 i}(t), \ldots, x_{N i}(t)\right)^{T}, \quad \tilde{e}_{i}(t)=\left(e_{1 i}(t), e_{2 i}(t), \ldots, e_{N i}(t)\right)^{T}, \\
H_{1}\left(\tilde{x}_{i}(t)\right)=\left(H_{1}\left(x_{1 i}(t)\right), H_{1}\left(x_{2 i}(t)\right), \ldots, H_{1}\left(x_{N i}(t)\right)\right)^{T}, \\
H_{2}\left(\tilde{x}_{i}(t)\right)=\left(H_{2}\left(x_{1 i}(t)\right), H_{2}\left(x_{2 i}(t)\right), \ldots, H_{2}\left(x_{N i}(t)\right)\right)^{T}, \quad \tilde{e}_{i}=\tilde{x}_{i}(t)-\tilde{s}_{i}(t) .
\end{gathered}
$$

Theorem 3.1. Suppose that Assumptions 2.7 and 2.8 hold, the adaptive coupling strength

$$
\sigma(t)=\widehat{\rho}(t)+\mu_{1}, \quad c(t)=\widehat{\eta}(t)+\mu_{2}
$$

where $\mu=\max \left(\mu_{1}, \mu_{2}\right), \mu_{1}>0, \mu_{2}>0$, and $\hat{\rho}(0)>0, \widehat{\eta}(0)>0$. If $\hat{\rho}(t), \widehat{\eta}(t)$ satisfies the following update law: $\dot{\hat{\rho}}(t)=-\sum_{i=1}^{N} e_{i}^{T}(t) e_{i}(t), \dot{\hat{\eta}}(t)=-\sum_{i=1}^{N}\left[e_{i}^{T}(t) e_{i}(t)+e_{i}^{T}(t-\tau(t)) e_{i}(t-\tau(t))\right]$ and the following condition hold:

$$
\mu_{1} \alpha \tilde{A}+\frac{1}{2(1-\varepsilon)} I_{N}+\frac{1}{2} \beta^{2} \mu_{2}^{2} B^{T} B<0
$$

then the controlled dynamical network (2.1) is synchronization.

Proof. Construct the following Lyapunov function:

$$
\begin{aligned}
V(t)= & \frac{1}{2} \sum_{i=1}^{N} e_{i}^{T}(t) e_{i}(t)+\frac{\theta}{(1-\varepsilon)} \sum_{i=1}^{N} \int_{t-\tau(t)}^{t} e_{i}^{T}(\xi) e_{i}(\xi) d \xi \\
& +\frac{1}{2(1-\varepsilon)} \sum_{j=1}^{n} \int_{t-\tau(t)}^{t} \tilde{e}_{j}^{T}(\xi) \tilde{e}_{j}(\xi) d \xi+\frac{\tilde{\rho}^{2}}{2 \beta_{1}}+\frac{\tilde{\eta}^{2}}{2 \beta_{2}}
\end{aligned}
$$

where $\dot{\tilde{\rho}}(t)=-\beta_{1} \sum_{i=1}^{N} e_{i}^{T}(t) e_{i}(t), \dot{\tilde{\eta}}(t)=-\beta_{2} \sum_{i=1}^{N}\left[e_{i}^{T}(t) e_{i}(t)+e_{i}^{T}(t-\tau(t)) e_{i}(t-\tau(t))\right], \tilde{\rho}(t)=$ $(\gamma+(\theta /(1-\varepsilon)))+\alpha \lambda_{1} \widehat{\rho}(t), \tilde{\eta}(t)=(1 / 2) \beta \lambda_{2} \widehat{\eta}(t), \lambda_{1}=\max \left(\left|\lambda_{1}(A)\right|,\left|\lambda_{2}(A)\right|, \ldots,\left|\lambda_{N}(A)\right|\right), \beta_{1}=\alpha \lambda_{1}$, 
$\lambda_{2}=\max \left(\left|\lambda_{1}(B)\right|,\left|\lambda_{2}(B)\right|, \ldots,\left|\lambda_{N}(B)\right|\right)$, and $\beta_{2}=(1 / 2) \beta \lambda_{2}$. In virtue of Assumptions 2.7 and 2.8 , the time derivative of $V(t)$ along the trajectory of system (2.4) is derived by

$$
\begin{aligned}
& \dot{V}(t)=\sum_{i=1}^{N} e_{i}^{T}(t)\left(\tilde{f}\left(t, x_{i}(t), x_{i}(t-\tau(t))\right)+\sigma(t) \sum_{j=1}^{N} a_{i j} H_{1}\left(x_{j}(t)\right)+c(t) \sum_{j=1}^{N} b_{i j} H_{2}\left(x_{i}(t-\tau(t))\right)-u_{i}(t)\right) \\
& +\frac{\theta}{(1-\varepsilon)} \sum_{i=1}^{N}\left(e_{i}^{T}(t) e_{i}(t)-(1-\dot{\tau}(t)) e_{i}^{T}(t-\tau(t)) e_{i}(t-\tau(t))\right) \\
& +\frac{1}{2(1-\varepsilon)} \sum_{j=1}^{n}\left(\tilde{e}_{j}^{T}(t) \tilde{e}_{j}(t)-(1-\dot{\tau}(t)) \tilde{e}_{j}^{T}(t-\tau(t)) \tilde{e}_{j}(t-\tau(t))\right)+\tilde{\rho}(t) \dot{\hat{\rho}}(t)+\tilde{\eta}(t) \dot{\hat{\eta}}(t) \\
& =\sum_{i=1}^{N} e_{i}^{T}(t) \tilde{f}\left(t, x_{i}(t), x_{i}(t-\tau(t))\right)+\sum_{i=1}^{N} e_{i}^{T}(t) \sigma(t) \sum_{j=1}^{N} a_{i j} H_{1}\left(x_{j}(t)\right) \\
& +\sum_{i=1}^{N} e_{i}^{T}(t) c(t) \sum_{j=1}^{N} b_{i j} H_{2}\left(x_{j}(t-\tau(t))\right)-\sum_{i=1}^{l} k_{i} e_{i}^{T}(t)\left(H_{1}\left(x_{i}(t)\right)-H_{1}(s(t))\right) \\
& +\frac{\theta}{(1-\varepsilon)} \sum_{i=1}^{N} e_{i}^{T}(t) e_{i}(t)-\frac{\theta(1-\dot{\tau}(t))}{(1-\varepsilon)} \sum_{i=1}^{N} e_{i}^{T}(t-\tau(t)) e_{i}(t-\tau(t)) \\
& +\frac{1}{2(1-\varepsilon)} \sum_{j=1}^{n} \tilde{e}_{j}^{T}(t) \tilde{e}_{j}(t)-\frac{1-\dot{\tau}(t)}{2(1-\varepsilon)} \sum_{j=1}^{n} \tilde{e}_{j}^{T}(t-\tau(t)) \tilde{e}_{j}(t-\tau(t))+\tilde{\rho}(t) \dot{\hat{\rho}}(t)+\tilde{\eta}(t) \dot{\hat{\eta}}(t) \\
& \leq \sum_{i=1}^{N}\left[\gamma e_{i}(t)^{T} e_{i}(t)+\theta e_{i}^{T}(t-\tau(t)) e_{i}(t-\tau(t))\right]+\sum_{i=1}^{N} e_{i}^{T}(t) \widehat{\rho}(t) \sum_{j=1}^{N} a_{i j} H_{1}\left(x_{j}(t)\right) \\
& +\mu_{1} \sum_{i=1}^{N} e_{i}^{T}(t) \sum_{j=1}^{N} a_{i j} H_{1}\left(x_{j}(t)\right)+\sum_{i=1}^{N} e_{i}^{T}(t) \widehat{\eta}(t) \sum_{j=1}^{N} b_{i j} H_{2}\left(x_{j}(t-\tau(t))\right) \\
& +\mu_{2} \sum_{i=1}^{N} e_{i}^{T}(t) \sum_{j=1}^{N} b_{i j} H_{2}\left(x_{j}(t-\tau(t))\right)-\sum_{i=1}^{l} k_{i} e_{i}^{T}(t)\left(H_{1}\left(x_{i}(t)\right)-H_{1}(s(t))\right) \\
& +\frac{\theta}{(1-\varepsilon)} \sum_{i=1}^{N} e_{i}^{T}(t) e_{i}(t)-\frac{\theta(1-\dot{\tau}(t))}{(1-\varepsilon)} \sum_{i=1}^{N} e_{i}^{T}(t-\tau(t)) e_{i}(t-\tau(t)) \\
& +\frac{1}{2(1-\varepsilon)} \sum_{j=1}^{n} \widetilde{e}_{j}^{T}(t) \tilde{e}_{j}(t)-\frac{1-\dot{\tau}(t)}{2(1-\varepsilon)} \sum_{j=1}^{n} \tilde{e}_{j}^{T}(t-\tau(t)) \tilde{e}_{j}(t-\tau(t))+\tilde{\rho}(t) \dot{\hat{\rho}}(t)+\widetilde{\eta}(t) \dot{\hat{\eta}}(t) \\
& \leq \gamma \sum_{i=1}^{N} e_{i}(t)^{T} e_{i}(t)+\theta \sum_{i=1}^{N} e_{i}(t-\tau(t))^{T} e_{i}(t-\tau(t))+\mu_{1} \sum_{i=1}^{n} \tilde{e}_{j}^{T}(t) \widetilde{A}\left(H_{1}\left(\tilde{x}_{j}(t)\right)-H_{1}(\widetilde{s}(t))\right)
\end{aligned}
$$




$$
\begin{aligned}
& +\mu_{2} \sum_{i=1}^{n} \tilde{e}_{j}^{T}(t) B\left(H_{2}\left(\tilde{x}_{j}(t-\tau(t))\right)-H_{2}\left(\widetilde{s}_{j}(t-\tau(t))\right)\right) \\
& +\frac{\theta}{(1-\varepsilon)} \sum_{i=1}^{N} e_{i}^{T}(t) e_{i}(t)-\frac{\theta(1-\dot{\tau}(t))}{(1-\varepsilon)} \sum_{i=1}^{N} e_{i}^{T}(t-\tau(t)) e_{i}(t-\tau(t)) \\
& +\frac{1}{2(1-\varepsilon)} \sum_{j=1}^{n} \tilde{e}_{j}^{T}(t) \tilde{e}_{j}(t)-\frac{1-\dot{\tau}(t)}{2(1-\varepsilon)} \sum_{j=1}^{n} \tilde{e}_{j}^{T}(t-\tau(t)) \tilde{e}_{j}(t-\tau(t))+\tilde{\rho}(t) \dot{\hat{\rho}}(t)+\tilde{\eta}(t) \dot{\hat{\eta}}(t) \\
& +\sum_{i=1}^{N} e_{i}^{T}(t) \widehat{\rho}(t) \sum_{j=1}^{N} a_{i j} H_{1}\left(x_{j}(t)\right)+\sum_{i=1}^{N} e_{i}^{T}(t) \widehat{\eta}(t) \sum_{j=1}^{N} b_{i j} H_{2}\left(x_{j}(t-\tau(t))\right) \\
& \leq\left(\gamma+\frac{\theta}{(1-\varepsilon)}\right) \sum_{i=1}^{N} e_{i}(t)^{T} e_{i}(t)+\mu_{1} \alpha \sum_{j=1}^{n} \tilde{e}_{j}^{T}(t) \tilde{A} \widetilde{e}_{j}(t)+\mu_{2} \beta \sum_{j=1}^{n} \tilde{e}_{j}^{T}(t) B \tilde{e}_{j}(t-\tau(t)) \\
& +\frac{1}{2(1-\varepsilon)} \sum_{j=1}^{n} \tilde{e}_{j}^{T}(t) \tilde{e}_{j}(t)-\frac{1}{2} \sum_{j=1}^{n} \tilde{e}_{j}^{T}(t-\tau(t)) \tilde{e}_{j}(t-\tau(t))+\tilde{\rho}(t) \dot{\hat{\rho}}(t)+\widetilde{\eta}(t) \dot{\hat{\eta}}(t) \\
& +\sum_{i=1}^{N} e_{i}^{T}(t) \widehat{\rho}(t) \sum_{j=1}^{N} a_{i j} H_{1}\left(x_{j}(t)\right)+\sum_{i=1}^{N} e_{i}^{T}(t) \widehat{\eta}(t) \sum_{j=1}^{N} b_{i j} H_{2}\left(x_{j}(t-\tau(t))\right) .
\end{aligned}
$$

$$
\begin{aligned}
& \text { Let } e(t)=\left(e_{1}^{T}(t), e_{2}^{T}(t), \ldots, e_{N}^{T}(t)\right)^{T} \in R^{n N} \text {, } \\
& \dot{V}(t) \leq\left(\gamma+\frac{\theta}{(1-\varepsilon)}\right) \sum_{i=1}^{N} e_{i}(t)^{T} e_{i}(t)+\mu_{1} \alpha \sum_{j=1}^{n} \tilde{e}_{j}^{T}(t) \tilde{A} \tilde{e}_{j}(t)+\mu_{2} \beta \sum_{j=1}^{n} \tilde{e}_{j}^{T}(t) B \tilde{e}_{j}(t-\tau(t)) \\
& +\frac{1}{2(1-\varepsilon)} \sum_{j=1}^{n} \tilde{e}_{j}^{T}(t) \tilde{e}_{j}(t)-\frac{1}{2} \sum_{j=1}^{n} \tilde{e}_{j}^{T}(t-\tau(t)) \tilde{e}_{j}(t-\tau(t))+\tilde{\rho}(t) \dot{\hat{\rho}}(t)+\tilde{\eta}(t) \dot{\hat{\eta}}(t) \\
& +\alpha \widehat{\rho}(t) e^{T}(t) A e(t)+\beta \widehat{\eta}(t) e^{T}(t) B e(t-\tau(t)) \\
& \leq \mu_{1} \alpha \sum_{j=1}^{n} \tilde{e}_{j}^{T}(t) \tilde{A} \widetilde{e}_{j}(t)+\mu_{2} \beta \sum_{j=1}^{n} \tilde{e}_{j}^{T}(t) B \widetilde{e}_{j}(t-\tau(t))+\frac{1}{2(1-\varepsilon)} \sum_{j=1}^{n} \tilde{e}_{j}^{T}(t) \tilde{e}_{j}(t) \\
& -\frac{1}{2} \sum_{j=1}^{n} \widetilde{e}_{j}^{T}(t-\tau(t)) \tilde{e}_{j}(t-\tau(t))+\widetilde{\eta}(t) \dot{\hat{\eta}}(t)+\frac{1}{2} \beta \lambda_{2} \widehat{\eta}(t)\left(e^{T}(t) e(t)+e^{T}(t-\tau(t)) e(t-\tau(t))\right)
\end{aligned}
$$


Mathematical Problems in Engineering

$$
\begin{aligned}
\leq & \mu_{1} \alpha \sum_{j=1}^{n} \tilde{e}_{j}^{T}(t) \tilde{A} \tilde{e}_{j}(t)+\mu_{2} \beta \sum_{j=1}^{n} \tilde{e}_{j}^{T}(t) B \tilde{e}_{j}(t-\tau(t))+\frac{1}{2(1-\varepsilon)} \sum_{j=1}^{n} \tilde{e}_{j}^{T}(t) \tilde{e}_{j}(t) \\
& -\frac{1}{2} \sum_{j=1}^{n} \tilde{e}_{j}^{T}(t-\tau(t)) \tilde{e}_{j}(t-\tau(t)) \\
\leq & \sum_{j=1}^{n} \tilde{e}_{j}^{T}(t)\left(\mu_{1} \alpha \tilde{A}+\frac{1}{2(1-\varepsilon)} I\right) \tilde{e}_{j}(t)+\beta \mu_{2} \sum_{j=1}^{n} \tilde{e}_{j}^{T}(t) B \tilde{e}_{j}(t-\tau(t)) \\
& -\frac{1}{2} \sum_{j=1}^{n} \tilde{e}_{j}^{T}(t-\tau(t)) \tilde{e}_{j}(t-\tau(t)) \\
\leq & \sum_{j=1}^{n}\left(\tilde{e}_{j}^{T}(t), \tilde{e}_{j}^{T}(t-\tau(t))\right) Z_{j}\left(\tilde{e}_{j}(t), \tilde{e}_{j}(t-\tau(t))\right)
\end{aligned}
$$

where

$$
Z_{j}=\left(\begin{array}{cc}
\mu_{1} \alpha \tilde{A}+\frac{1}{2(1-\varepsilon)} I_{N} & \frac{1}{2} \beta \mu_{2} B \\
\frac{1}{2} \beta \mu_{2} B^{T} & -\frac{1}{2} I_{N}
\end{array}\right)
$$

It follows from Lemma 2.5 that if

$$
\mu_{1} \alpha \tilde{A}+\frac{1}{2(1-\varepsilon)} I_{N}+\frac{1}{2} \beta^{2} \mu_{2}^{2} B^{T} B<0
$$

we obtain $\dot{V}(t)<0$. The proof is completed.

Remark 3.2. Compared with the other control methods in the literature, pinning controller is relatively simple and is easy to implement. As we know now, the real-world complex networks normally have a large number of nodes. Therefore, it is usually difficult to control a complex network by adding the controllers to all nodes. To reduce the number of the controllers, a natural approach is to control a complex network by pinning part of nodes. In this paper, we designed controllers to ensure that the special networks could get synchronization. The pinning nodes can be randomly selected. It indeed provides some new insights for the future practical engineering design. 
Remark 3.3. Synchronization criteria have been given in Theorem 3.1. Compared with some similar designs, our pinning adaptive controllers are very simple. In particular, the synchronization criteria are independent of time delays.

\section{Illustrative Examples}

In this section, a numerical example will be given to demonstrate the validity of the synchronization criteria obtained in the previous sections. Considering the followingnetwork:

$$
\begin{aligned}
\dot{x}_{i}(t)= & f\left(t, x_{i}(t), x_{i}(t-\tau(t))\right)+\sigma(t) \sum_{j=1}^{N} a_{i j} H_{1}\left(x_{j}(t)\right) \\
& +c(t) \sum_{j=1}^{N} b_{i j} H_{2}\left(x_{j}(t-\tau(t))\right)-u_{i}(t), \quad i=1,2, \ldots, l, \\
\dot{x}_{i}(t)= & f\left(t, x_{i}(t), x_{i}(t-\tau(t))\right)+\sigma(t) \sum_{j=1}^{N} a_{i j} H_{1}\left(x_{j}(t)\right) \\
& +c(t) \sum_{j=1}^{N} b_{i j} H_{2}\left(x_{j}(t-\tau(t))\right), \quad i=l+1, l+2 \cdots N,
\end{aligned}
$$

where $f\left(t, x_{i}(t), x_{i}(t-\tau(t))\right)=D x_{i}(t)+h_{1}\left(x_{i}(t)\right)+h_{2}\left(x_{i}(t-\tau(t))\right)$ here $\tau(t)=e^{t} / 100\left(1+e^{t}\right)$, $x_{i}(t)=\left(x_{i 1}(t), x_{i 2}(t), x_{i 3}(t)\right)^{T}, h_{1}\left(x_{i}\right)=\left(0,-x_{i 1} x_{i 3}, x_{i 1} x_{i 2}\right)^{T}, h_{2}\left(x_{i}\right)=\left(0,0.2 x_{i 2}, 0\right)^{T}, \mu=5, N=$ $4, l=1, H_{1}(x)=\sin x+2 x, H_{2}(x)=\cos x+3 x$, and

$$
\begin{gathered}
D=\left[\begin{array}{ccc}
-10 & 10 & 0 \\
28 & -1 & 0 \\
0 & 0 & -\frac{8}{3}
\end{array}\right], \\
A=B=\left[\begin{array}{cccc}
-1 & 1 & 0 & 0 \\
1 & -3 & 1 & 1 \\
0 & 1 & -2 & 1 \\
0 & 1 & 1 & -2
\end{array}\right] .
\end{gathered}
$$

\section{Conclusions}

The problems of synchronization and pinning control for the nonlinearly coupled complex networks with time-varying coupling delay and time-varying delay in dynamical nodes are 


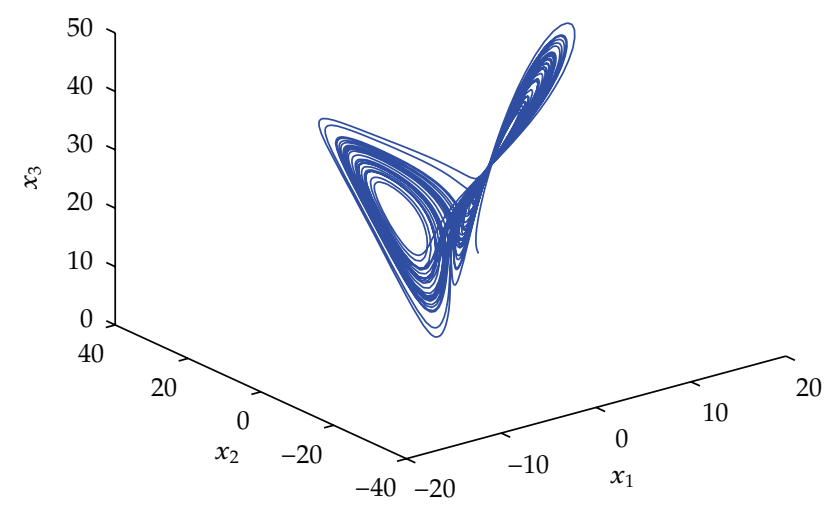

Figure 1: The chaotic behavior of time-delayed Lorenz system.
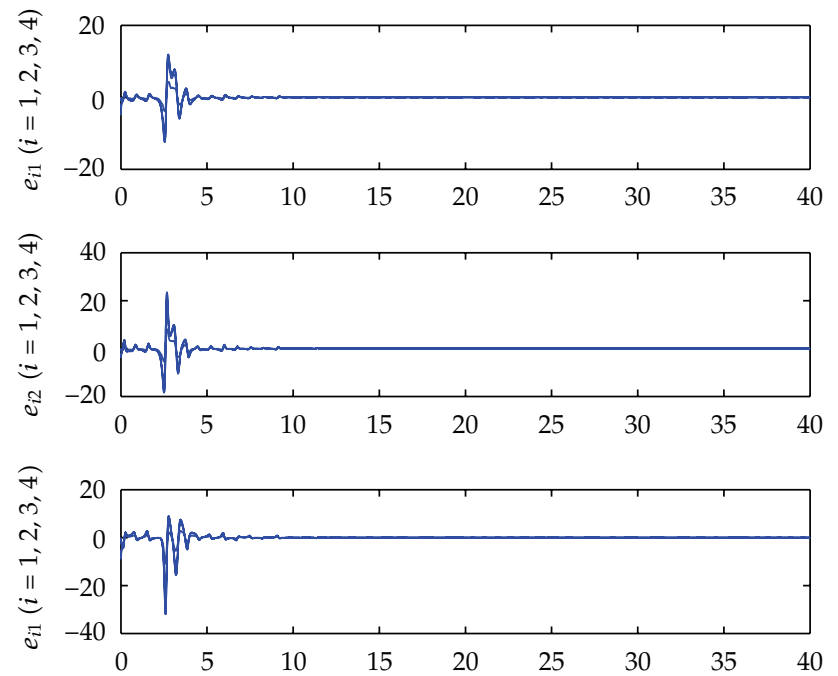

Figure 2: Time evolution of the synchronization errors.

investigated. It is shown that synchronization can be realized via adjusting time-varying coupling strengths. The study showed that the use of simple control law helps to derive sufficient criteria which ensure that the synchronization of the network model is derived. In addition numerical simulations were performed to verify the effectiveness of the theoretical results. Compared with existing results, our synchronization is still very useful when the existing methods become invalid (Figures 1 and 2).

\section{Acknowledgments}

Research is partially supported by the National Nature Science Foundation of China (no. 70871056) and by the Six Talents Peak Foundation of Jiangsu Province. The authors are also grateful to Miss Mary Opokua Ansong, at the Department of Computer Science and Technology, Jiangsu University, for making time to go through this work. 


\section{References}

[1] F. Sorrentino, "Effects of the network structural properties on its controllability," Chaos, vol. 17, no. 3, Article ID 033101, 2007.

[2] M. Porfiri and M. D. Bernardo, "Criteria for global pinning-controllability of complex networks," Automatica, vol. 44, no. 12, pp. 3100-3106, 2008.

[3] S. Y. Chen, W. Huang, and C. Cattani, "Traffic dynamics on complex networks: a survey," Mathematical Problems in Engineering, vol. 2012, Article ID 732698, 23 pages, 2012.

[4] S. Chen, H. Tong, and C. Cattani, "Markov models for image labeling," Mathematical Problems in Engineering, vol. 2012, Article ID 814356, 18 pages, 2012.

[5] F. Sorrentino, M. Di Bernardo, F. Garofalo, and G. Chen, “Controllability of complex networks via pinning," Physical Review E, vol. 75, no. 4, Article ID 046103, 2007.

[6] S. N. Dorogovtsev and J. F. F. Mendes, Evolution of Networks: From Biological Nets to the Internet and WWW, Oxford University Press, Oxford, UK, 2003.

[7] P. M. Gade, "Synchronization of oscillators with random nonlocal connectivity," Physical Review E, vol. 54, no. 1, pp. 64-70, 1996.

[8] J. Lu, D. W. C. Ho, and M. Liu, "Globally exponential synchronization in an array of asymmetric coupled neural networks," Physics Letters, Section A, vol. 369, no. 5-6, pp. 444-451, 2007.

[9] H. Liu, J. Chen, J. A. Lu, and M. Cao, "Generalized synchronization in complex dynamical networks via adaptive couplings," Physica A, vol. 389, no. 8, pp. 1759-1770, 2010.

[10] M. G. Rosenblum, A. S. Pikovsky, and J. Kurths, "Phase synchronization of chaotic oscillators," Physical Review Letters, vol. 76, no. 11, pp. 1804-1807, 1996.

[11] M. G. Rosenblum, A. S. Pikovsky, and J. Kurths, "From phase to lag synchronization in coupled chaotic oscillators," Physical Review Letters, vol. 78, no. 22, pp. 4193-4196, 1997.

[12] R. Zhang, Y. Yang, Z. Xu, and M. Hu, "Function projective synchronization in drive-response dynamical network," Physics Letters, Section A, vol. 374, no. 30, pp. 3025-3028, 2010.

[13] S. Zheng, "Adaptive-impulsive control of the projective synchronization in drive-response complex dynamical networks with time-varying coupling," Mathematical Problems in Engineering, vol. 2012, Article ID 501843, 12 pages, 2012.

[14] J. Zhou, J. Lu, and J. Lü, "Adaptive synchronization of an uncertain complex dynamical network," Institute of Electrical and Electronics Engineers. Transactions on Automatic Control, vol. 51, no. 4, pp. 652656, 2006.

[15] L. Pan, W. Zhou, J. Fang, and D. Li, “Synchronization and anti-synchronization of new uncertain fractional-order modified unified chaotic systems via novel active pinning control," Communications in Nonlinear Science and Numerical Simulation, vol. 15, no. 12, pp. 3754-3762, 2010.

[16] S. Zheng and Q.S. Bi, "Synchronization analysis of complex dynamical networks with delayed and non-delayed coupling based on pinning control," Physica Scripta, vol. 84, Article ID 025008, 2011.

[17] G. Liu and W. Ding, "Impulsive synchronization for a chaotic system with channel time-delay," Communications in Nonlinear Science and Numerical Simulation, vol. 16, no. 2, pp. 958-965, 2011.

[18] L. X. Guo, M. F. Hu, and Z. Y. Xu, "Impulsive synchronization and control of directed transport in chaotic ratchets," Chinese Physics B, vol. 19, no. 2, Article ID 020512, 2010.

[19] S. Zheng, "Adaptive-impulsive projective synchronization of drive-response delayed complex dynamical networks with time-varying coupling," Nonlinear Dynamics.. In press.

[20] S. G. Wang and H. X. Yao, "Impulsive synchronization of two coupled complex networks with timedelayed dynamical nodes," Chinese Physics B, vol. 20, no. 9, Article ID 090513, 2011.

[21] A. A. Syntetos and J. E. Boylan, "On the stock control performance of intermittent demand estimators," International Journal of Production Economics, vol. 103, no. 1, pp. 36-47, 2006.

[22] S. M. Cai, J. Hao, Q. He, and Z. Liu, "Exponential synchronization of complex delayed dynamical networks via pinning periodically intermittent control," Physics Letters, Section A, vol. 375, no. 19, pp. 1965-1971, 2011.

[23] T. Liu, J. Zhao, and D. J. Hill, "Synchronization of complex delayed dynamical networks with nonlinearly coupled nodes," Chaos, Solitons and Fractals, vol. 40, no. 3, pp. 1506-1519, 2009.

[24] W. Guo, F. Austin, S. Chen, and W. Sun, "Pinning synchronization of the complex networks with nondelayed and delayed coupling," Physics Letters, Section A, vol. 373, no. 17, pp. 1565-1572, 2009.

[25] X. F. Wang and G. Chen, "Pinning control of scale-free dynamical networks," Physica A, vol. 310, no. 3-4, pp. 521-531, 2002.

[26] T. Chen, X. Liu, and W. Lu, "Pinning complex networks by a single controller," IEEE Transactions on Circuits and Systems. I, vol. 54, no. 6, pp. 1317-1326, 2007. 
[27] W. W. Yu, G. Chen, and J. H. Lue, "On pinning synchronization of complex dynamical networks," Automatica, vol. 45, no. 2, pp. 429-435, 2009.

[28] W. W. Yu, J. Cao, and J. Lü, “Global synchronization of linearly hybrid coupled networks with timevarying delay," SIAM Journal on Applied Dynamical Systems, vol. 7, no. 1, pp. 108-133, 2008.

[29] W. Lu and T. Chen, "New approach to synchronization analysis of linearly coupled ordinary differential systems," Physica D., vol. 213, no. 2, pp. 214-230, 2006.

[30] Z. Zuo, C. Yang, and Y. Wang, "A unified framework of exponential synchronization for complex networks with time-varying delays," Physics Letters, Section A, vol. 374, no. 19-20, pp. 1989-1999, 2010.

[31] J. Zhou and T. Chen, "Synchronization in general complex delayed dynamical networks," IEEE Transactions on Circuits and Systems. I, vol. 53, no. 3, pp. 733-744, 2006.

[32] C. Hu, J. Yu, H. Jiang, and Z. Teng, "Synchronization of complex community networks with nonidentical nodes and adaptive coupling strength," Physics Letters, Section A, vol. 375, no. 5, pp. 873-879, 2011. 


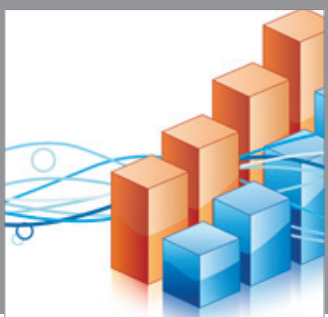

Advances in

Operations Research

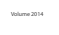

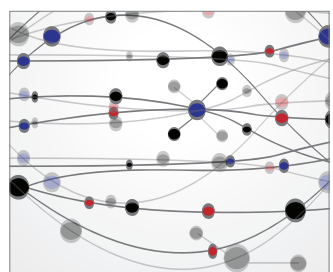

\section{The Scientific} World Journal
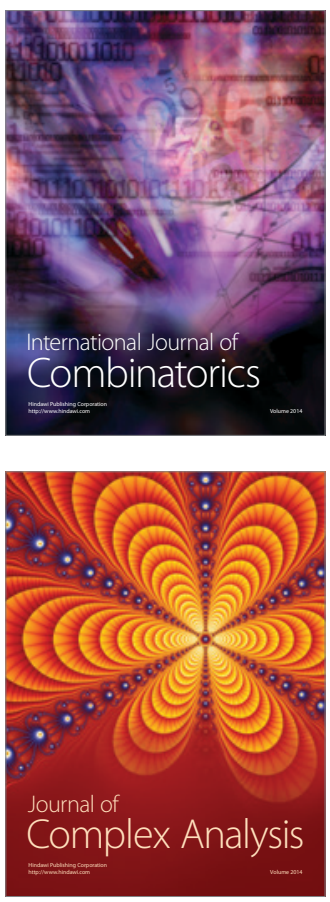

International Journal of

Mathematics and

Mathematical

Sciences
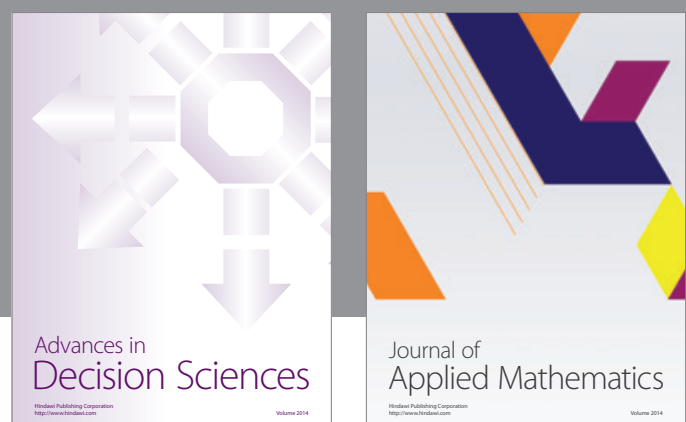

Journal of

Applied Mathematics
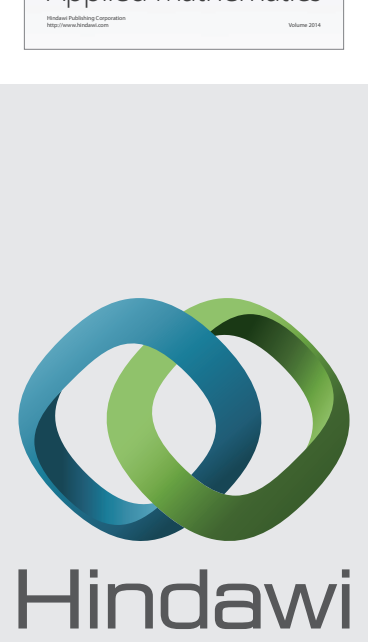

Submit your manuscripts at http://www.hindawi.com
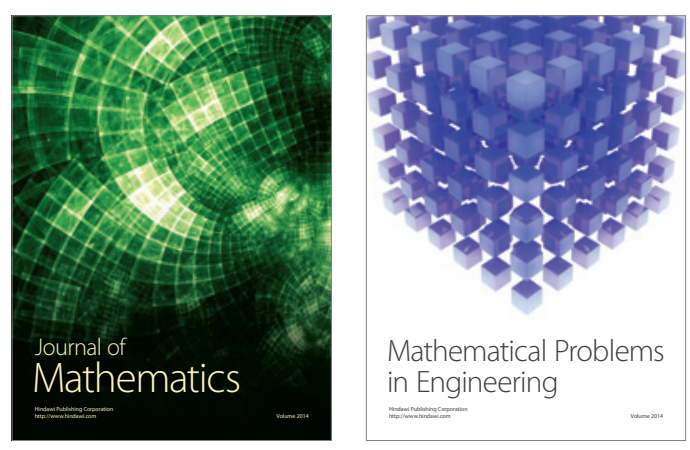

Mathematical Problems in Engineering
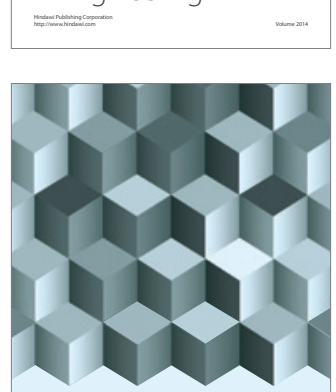

Journal of

Function Spaces
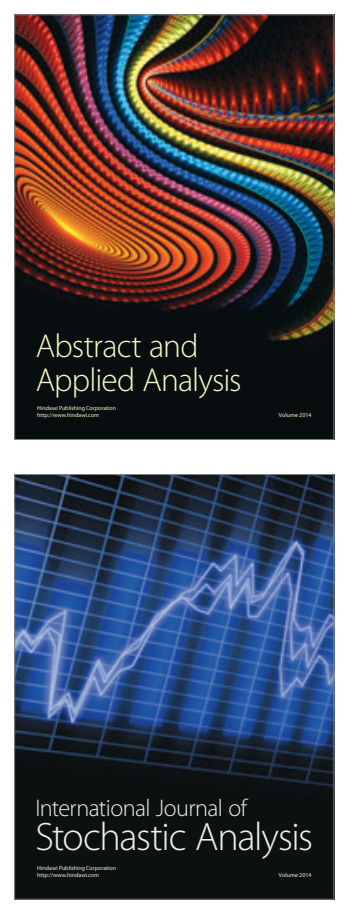

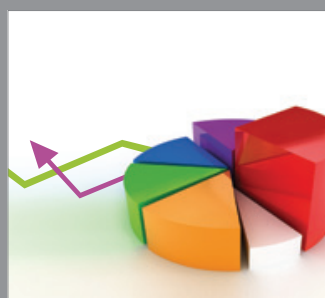

ournal of

Probability and Statistics

Promensencen
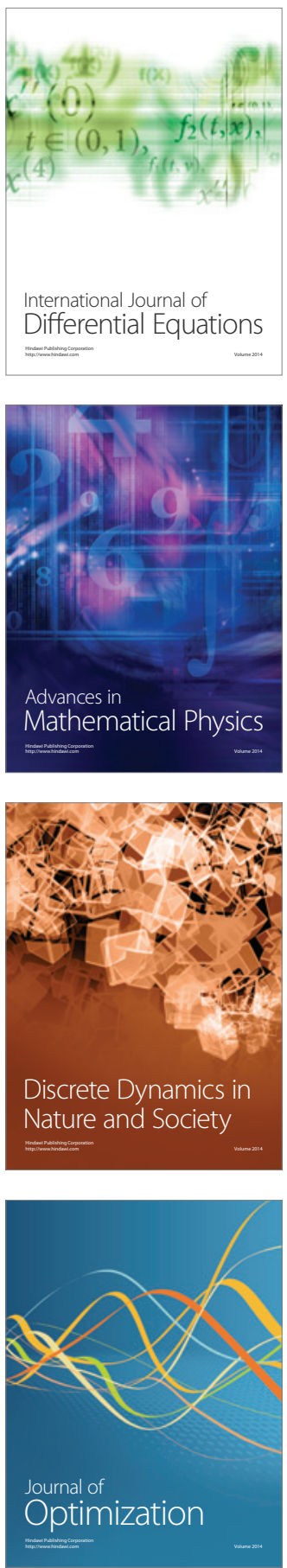